\title{
Isolating a Minimum Radius of the Universe Consistent with the Production of at Least 1 Unit of Entropy, at the Start of Inflation
}

\author{
Andrew Walcott Beckwith \\ Physics Department, College of Physics, Chongqing University, Chongqing China \\ Email:Rwill9955b@gmail.com, abeckwith@uh.edu
}

How to cite this paper: Beckwith, A.W. (2017) Isolating a Minimum Radius of the Universe Consistent with the Production of at Least 1 Unit of Entropy, at the Start of Inflation. Journal of High Energy Physics, Gravitation and Cosmology, 3, 461-466. https://doi.org/10.4236/jhepgc.2017.33036

Received: March 22, 2017

Accepted: July 28, 2017

Published: July 31, 2017

Copyright $\odot 2017$ by author and Scientific Research Publishing Inc. This work is licensed under the Creative Commons Attribution International License (CC BY 4.0). http://creativecommons.org/licenses/by/4.0/

\begin{abstract}
We begin by examining a general expression of entropy, and its links to a minimum radius of the universe. We derive an expression for the production of at least 1 unit of entropy, which translates to a value of Planck length in radii to 1000 times Planck radii, for the quantum bubble of space-time which depends upon, of all things, the initial Hubble expansion rate value. If the Hubble parameter has the value of $10^{\wedge} 19 \mathrm{GeV}$, we see a minimum radial length of the Universe of about 1 billion times Planck length. If the Hubble parameter is of $10^{\wedge} 19 \mathrm{GeV}$, the minimum radial length of the universe would be about one Planck length, which is surprising to put it mildly. The higher the initial temperate is, up to a point, the more likely the initial entropy is closer to the Causal barrier mentioned in an earlier publication by the author.
\end{abstract}

\section{Keywords}

Causal Barrier, Inflaton, Mininum Entropy (Non Zero), Initial Radii (of Universe)

\section{Introduction}

We begin with the expression given in [1] [2] [3], with $F$ the free energy, and $S$ the entropy, so that

$$
\begin{aligned}
& F=-k_{B} T \ln Z \\
& S=-\left(\frac{\partial F}{\partial T}\right)_{V, N} \sim k_{B} T \ln Z \\
& \propto n \text { (entropy-count) }
\end{aligned}
$$

The end result is that we will approximate the entropy count as given by the last line of Equation (1) so that we can refer to an article in [4] for which there 
exists a critical Hubble parameter, $H_{I}$ for which we have by [1] a $H_{I}=10^{10} \mathrm{GeV}$ value that will be shown to have $n$ (entropy-count) $\sim 1$, with a radius of the universe of about 1000 times Planck length. Also, where if we have instead, $H_{I}=10^{18} \mathrm{GeV}$, we have $n$ (entropy-count) $\sim 1$ with a radius of the Universe of about one Planck length. After this is done, we will then comment upon the value of the square of the average inflaton value given by [5]. Where the square, of the inflaton, is given as follows, with a single inflaton given in Equation (2) as follows:

$$
\begin{aligned}
\left\langle\phi^{2}\right\rangle & =\frac{3 H_{I}^{2}}{8 \pi^{2} m^{2}} \cdot\left(1-\exp \left[-\frac{2 m^{2}}{3 H_{I}} \cdot\left(t-t_{0}\right)\right]\right) \\
& \approx \frac{3 H_{I}^{3} \cdot\left(t-t_{0}\right)}{8 \pi^{2}} \text { if }\left(t-t_{0}\right) \ll 3 H_{I} / m^{2} \\
\& \phi & =\sqrt{\left\langle\phi^{2}\right\rangle}=\frac{H_{I}}{2 \pi} \cdot \sqrt{H_{I} \Delta t}
\end{aligned}
$$

With the substitution of $m$ as the mass of a graviton, as given in [6], i.e. about $10^{\wedge}-62$ grams, the inequality leading to a graviton mass induced behavior of the inflaton which we will comment upon fully while making use of $\Delta t$ that is discussed in [7] [8]. In doing so, if one wants to be close to a Pre-Planckian length and time step, the preference would use $H_{I}=10^{18} \mathrm{GeV}$, which then gets to the issue of interpretation of what to make of the following, from [9], i.e. if $g^{*}$ refers to initial degrees of freedom, then we will interpret several different cases for Equation (3) below, with different mass scale ideas in, and different initial temperature scenarios.

$$
H_{\text {Early-Universe }} \sim 1.66 \cdot \sqrt{g^{*}} \cdot \frac{T_{\text {Early-Universe }}}{M_{\text {mass-scale }}}
$$

We will discuss all this and more in the subsequent analysis. Our final points will be using a comment from Rudin, as to outer measures [10] and its relationship to the causal structure brought up in [11] and [12].

\section{The Basic Analysis to Consider}

What we are looking at first of all, is if Equation (1) is true, and Entropy is a counting algorithm, which is not so farfetched, then by use of [4] for entropy, and $H_{I}$ as well as [3] for $S$ (entropy) $\propto n$ (particle count) then

$$
\begin{aligned}
& S=-\left(\frac{\partial F}{\partial T}\right)_{V, N} \sim k_{B} T \ln Z \\
& S \propto n(\text { entropy-count }) \sim \frac{H_{I}^{3}}{180 \pi} \cdot[\text { Volume (space-time) }]
\end{aligned}
$$

Then, we have that

$$
\begin{aligned}
S & \propto n(\text { entropy-count }) \sim \frac{H_{I}^{3}}{180 \pi} \cdot[\text { Volume }(\text { space-time })] \\
& \sim \frac{H_{I}^{3}}{180 \pi} \cdot\left[\beta^{3} \times l_{\text {Planck }}^{3}\right]
\end{aligned}
$$


We then will pick the smallest possible entropy value of $n$ as equal to 1 . Then what we are looking at is

$$
\beta \sim\left[\frac{H_{I}^{3}}{180 \pi}\right]^{-1 / 3}
$$

We will look at two cases for our analysis, and the first one is with the [5] value of $H_{I}=10^{10} \mathrm{GeV}$.

\section{What If $H_{I}=10^{10} \mathrm{GeV}$ ? Consequences for Equation (6)}

Here we first normalize the $H_{I}=10^{10} \mathrm{GeV}$ with regards to the Planck Mass. The Planck Mass is [13]

$$
M_{\text {Planck }} \approx 4.341 \times 10^{-9} \mathrm{~kg}=2.435 \times 10^{18} \mathrm{GeV} / \mathrm{c}^{2}
$$

We will set $c=1 \mathrm{a}$, normalize the Planck mass to be $=1$. If so then we write

$$
H_{I}=10^{10} \mathrm{GeV} \underset{\text { Planck-Unit-Normalization,wtih } M_{\text {Planck } \rightarrow 1}}{\longrightarrow} \frac{10^{-8}}{2.435}
$$

If so then, we will have Equation (6) rendered to be

$$
\beta \sim\left[\frac{H_{I}^{3}}{180 \pi}\right]^{-1 / 3} \sim\left[\frac{180 \pi \times(2.435)^{3}}{10^{-24}}\right]^{1 / 3} \sim 10^{8} \times 2.435 \times 8.267 \sim 10^{8} \times 20.13 \sim 2 \times 10^{9}
$$

By inspection, it means in order to have Equation (6) of the order of magnitude of about 1 , or less, we need to look at

$$
H_{I}=10^{19} \mathrm{GeV} \underset{\text { Planck-Unit-Normalization,wth } M_{\text {Planck }} \rightarrow 1}{\longrightarrow} \frac{10^{1}}{2.435} \sim 4.106
$$

i.e. $H_{I}$ would be at least a billion times larger than $H_{I}=10^{10} \mathrm{GeV}$, in order to have Planck length of radii of the initial configuration of space time for at least 1 unit of entropy production. With the lower value of $H_{I}$ as specified in [5] we would have then an initial radii of Planck length times one billion for about 1 unit of entropy production of our analysis.

4. Consequences of $H_{I}=10^{19} \mathrm{GeV} \sim \frac{10^{1}}{2.435} \sim 4.106$ Instead of

$$
H_{I}=10^{10} \mathrm{GeV} \sim \frac{10^{-8}}{2.435}
$$

Going back to the Equation (3) it depends upon what we choose the Mass, in the denominator, to be, of which if it is Planck Mass, and we normalize that to 1 , then we have at the boundary of about a Planck length for a 1 entropy value, i.e. one count of a primordial particle, but at an insanely high initial temperature, of the order of Planck temperature of about $1.417 \times 10^{32}$ kelvin, or about $1.22 \times 10^{19}$ $\mathrm{GeV}$, for the given production of 1 unit of Planck mass. Note that Planck length is about, in simple units set about being $1.616229(38) \times 10^{-35} \mathrm{~m}$ so this means that the radii of the universe even with 


$$
\begin{aligned}
& H_{I}=10^{10} \mathrm{GeV} \underset{\text { Planck-Unit-Normalization,wth } M_{\text {Planck }} \rightarrow 1}{\longrightarrow} \frac{10^{-8}}{2.435} \\
& \Rightarrow \text { Radius (Universe) for } n \geq 1 \text { about } 10^{-26} \text { meters } \\
& H_{I}=10^{19} \mathrm{GeV} \underset{\text { Planck-Unit-Normalization,wth } M_{\text {Planck }} \rightarrow 1}{\longrightarrow} \frac{10^{1}}{2.435} \sim 4.106 \\
& \Rightarrow \text { Radius (Universe) for } n \geq 1 \text { about } 10^{-35} \text { meters }
\end{aligned}
$$

\section{Conclusion: Planck Radii in Length for Initial Configuration of Universe Could Lead to One Unit of Entropy Production. Consequences? Ultra High Temperatures. What Else?}

What we would have to go back to, then would be to review the ideas given in the document about the Dowker Structure of space-time as given in [12] and that would place a premium upon also understanding the role of the formulation of a causal boundary, as given in [11], where after this boundary, we would employ [11] after an initial radii of space-time, is traversed from an initial starting point given by what is written below.

$$
\left(\frac{\left.R_{c}\right|_{\text {initial }} \sim c \cdot \Delta t}{l_{\text {Planck }}}\right) \sim \vartheta(1)
$$

i.e. what we are asserting is that at Planck temperature, we are observing a convergence close to the value of where we may have the initiation of a Causal structure. If so, then is Entropy, initially created at the START pf causal structure, due to an opportune selection of a special unit of $\Delta t$ i.e. note that in [8] we did suggest that the formation of the arrow of time, so done, would be a precursor of entropy, i.e. our argument may in itself be a first principle proof that the arrow of time, as initially formed, is a precursor for at least 1 unit of entropy created, and that this would be commensurate with making sense of

$$
\phi=\sqrt{\left\langle\phi^{2}\right\rangle}=\frac{H_{I}}{2 \pi} \cdot \sqrt{H_{I} \Delta t}
$$

i.e. if we satisfy Equation (11), Equation (12), Equation (13) in the case of $H_{I}=10^{19} \mathrm{GeV} \sim \frac{10^{1}}{2.435} \sim 4.106$, we are also asserting that the formation of an Inflaton, according to [5], is in our estimation simultaneous with the formation of causal structure as indicated by Equation (12), as well as giving more inputs into the Padmadabhan model we used in [8] for the inflaton, i.e. [14], as well as the idea given by Corda as to the Gravity's breath suggestion he made. The author is aware of which the author finds quite pleasing to contemplate [15].

In doing so, in the formation of Causal structure, so long as it does not contravene the outer measure definition given in [10] and requirements linked toward getting Equation (11) satisfied, we are not in trouble, at least mathematically for the time being. We will be using the nonsingular approach pioneered by [16] and [17] and we should do our best to avoid problems in our analysis which may contradict the LIGO results of [18] and [19] [20], especially if each single 
count of $n$, as referenced to entropy is in common with gravitons. Also, our analysis should be further refined to take into account [20], which may be with initial doable instrument refinements. Even so, we also point to [21] as having relevance to early universe work in our future endeavors [22].

\section{Acknowledgements}

This work is supported in part by National Nature Science Foundation of China Grant No. 11375279.

\section{References}

[1] Reichl, L. (1980) A Modern Course in Statistical Physics. University of Texas Press, Austin, Texas, USA.

[2] Shankar, R. (2014) Fundamentals of Physics, Mechanics, Relativity, and Thermodynamics. Yale University Press, New Haven, Conn., USA.

[3] Ng, Y.J. (2008) Spacetime Foam: From Entropy and Holography to Infinite Statistics and Nonlocality. Entropy, 10, 441-461. https://doi.org/10.3390/e10040441

[4] Kiefer, C. (2000) Entropy of Gravitational Waves and Promordial Fluctuations. In: Garcia-Bellido, D.R. and Sharposhnikov, M., Eds., Cosmology and Particle Physics, CAPP 2000, Vebier, Vol. 555, AIP Conference Procedings in Physics, Melville, New York, 2001, USA, 499-504.

[5] Linde, A. (2016) The New Inflationary Universe Scenario. In: Jackliw, K.N., Weinbert, S.A. and Witten, E., Eds., Proceedings of the 1983 Shelter Island Conference on Quantum Field Theory and the Fundamental Problems of Physicswhich, Dover Publications, Mineola, New York, USA, 190-216.

[6] Zakharov, V.I. (1970) Linearized Gravitation Theory and the Graviton Mass. JETP Lett., 12, 312.

[7] Beckwith, A. How a Minimum Time Step Leads to a Causal Structure Used to Form Initial Entropy Production and High Frequency Gravitons, with 7 Subsequent Open Questions. http://vixra.org/abs/1703.0166

[8] Beckwith, A. How a Minimum Time Step Based in Pre Planckian Space-Time If Friedman Equation $\mathrm{H}$ Set Equal to Zero Leads to the Arrow of Time. http://vixra.org/abs/1702.0290

[9] Sarkar, U. (2008) Particle and Astro Particle Physics. Series in High Energy Physics and Gravitation. Taylor \& Francis, New York.

[10] Rudin, W. (1976) Principles of Mathematical Analysis. $3^{\text {rd }}$ Edition, McGraw Hill, and Copied by McGraw Hill Education (India) 2013, New Deli, India.

[11] Beckwith, A. (2017) How a Minimum Time Step Leads to the Construction of the Arrow of Time and the Formation of Initial Causal Structure in Space-Time. http://vixra.org/pdf/1702.0295v1.pdf

[12] Dowker, F. (2005) Causal Sets and the Deep Structure of Space-Time. https://arxiv.org/abs/grqc/0508109

[13] http://physics.nist.gov/cuu/Constants/index.html

[14] Padmanabhan, T. (2005) Understanding Our Universe: Current Status, and Open Issues. In: 100 Years of Relativity, Space-Time, Structure: Einstein and Beyond, World Scientific, Singapore, 175-204. http://arxiv.org/abs/gr-qc/0503107

[15] Corda, C. (2011) Primordial Gravity's Breath. EJTP, 9, 1-10. http://www.ejtp.com/articles/ejtpv9i26.pdf 
https://arxiv.org/abs/1110.1772

[16] Camara, C.S., de Garcia Maia, M.R., Carvalho, J.C. and Lima, J.A.S. (2004) Nonsingular FRW Cosmology and Non Linear Dynamics.

[17] Rovelli, C. and Vidotto, F. (2015) Covariant Loop Quantum Gravity. Cambridge University Press, Cambridge.

[18] Abbott, B.P., et al. (LIGO Scientific Collaboration and Virgo Collaboration) (2016) Observation of Gravitational Waves from a Binary Black Hole Merger. Physical Review Letters, 116, Article ID: 061102.

[19] Abbott, B.P., et al. (LIGO Scientific Collaboration and Virgo Collaboration) (2016) GW151226: Observation of Gravitational Waves from a 22-Solar-Mass Binary Black Hole Coalescence. Physical Review Letters, 116, Article ID: 241103.

[20] Abbott, B.P., et al. (2016) Tests of General Relativity with GW150914. https://arxiv.org/pdf/1602.03841.pdf

[21] Corda, C. (2009) Interferometric Detection of Gravitational Waves: The Definitive Test for General Relativity. International Journal of Modern Physics D, 18, 22752282. https://arxiv.org/abs/0905.2502 https://doi.org/10.1142/S0218271809015904

[22] Woods, R., Baker, R., Li, F., Stephenson, G., Davis, E. and Beckwith, A. (2011) A New Theoretical Technique for the Measurement of High-Frequency Relic Gravitational Waves. Journal of Modern Physics, 2, 498-518.

https://doi.org/10.4236/jmp.2011.26060

Submit or recommend next manuscript to SCIRP and we will provide best service for you:

Accepting pre-submission inquiries through Email, Facebook, LinkedIn, Twitter, etc. A wide selection of journals (inclusive of 9 subjects, more than 200 journals)

Providing 24-hour high-quality service

User-friendly online submission system

Fair and swift peer-review system

Efficient typesetting and proofreading procedure

Display of the result of downloads and visits, as well as the number of cited articles

Maximum dissemination of your research work

Submit your manuscript at: http://papersubmission.scirp.org/

Or contact jhepgc@scirp.org 